\title{
Four-dimensional conformal supergravity from AdS space
}

\author{
Vijay Balasubramanian, ${ }^{1, *}$ Eric Gimon, ${ }^{2, \dagger}$ Djordje Minic, ${ }^{3, \$}$ and Joachim Rahmfeld ${ }^{2, \S}$ \\ ${ }^{1}$ Jefferson Laboratory of Physics, Harvard University, Cambridge, Massachusetts 02138 \\ ${ }^{2}$ CIT-USC Center for Theoretical Physics, California Institute of Technology, Pasadena, California 91125 \\ ${ }^{3}$ CIT-USC Center for Theoretical Physics, Department of Physics and Astronomy, University of Southern California, \\ Los Angeles, California 90089-0484
}

(Received 9 August 2000; published 12 April 2001)

\begin{abstract}
Exploring the role of conformal theories of gravity in string theory, we show that the minimal $(N=2)$ gauged supergravities in five dimensions induce the multiplets and transformations of $N=1$ four-dimensional conformal supergravity on the spacetime boundary. $N=1$ Poincaré supergravity can be induced by explicitly breaking the conformal invariance via a radial cutoff in the 5D space. The AdS/CFT correspondence relates the maximal gauged supergravity in five dimensions to $N=4$ super Yang-Mills theory on the 4D spacetime boundary. In this context we show that the conformal anomaly of the gauge theory induces conformal gravity on the boundary of the space and that this theory, via the renormalization group, encapsulates the gravitational dynamics of the skin of asymptotically AdS spacetimes. Our results have several applications to the AdS/CFT correspondence and the Randall-Sundrum scenario.
\end{abstract}

DOI: 10.1103/PhysRevD.63.104009

PACS number(s): 04.65.+e, 11.25.Hf, 11.25.Mj

\section{INTRODUCTION}

The classic methods of Kaluza and Klein [1] are the conventional tools in supergravity for generating the dynamics of a lower dimensional space from a higher dimensional world with a compact factor. The light fields in the lower dimensions arise from fluctuations that solve massless wave equations on the internal space, and the symmetries governing their dynamics are derived by appropriate restriction from the higher dimensions. Recently, string theorists and phenomenologists have studied the physics of worlds that exist on branes or submanifolds embedded in a higher dimensional space. In the AdS/conformal field theory (CFT) [2,3] and Randall-Sundrum [4] contexts, the relevant 4-surface lies near or at the boundary of a five dimensional space which asymptotically has a negative cosmological constant. Such asymptotically anti-de Sitter (AdS) spaces arise naturally as solutions to $5 \mathrm{D}$ gauged supergravities $[5,6]$ or as the near horizon limits of string compactifications containing 3-branes [7]. One purpose of this article is to show in detail how $N=1$ supergravity is induced on such surfaces when the bulk theory enjoys $N=2$ supersymmetry. When the bulk is non-compact, the $N=1$ theory is conformal. ${ }^{1}$ Poincare supergravity can be regained by cutting off the bulk space to explicitly break conformality.

The AdS/CFT correspondence states that the classical action for an asymptotically AdS space, regulated by boundary counterterms (see, e.g., $[10,11])$ and treated as a functional of boundary data, is equal to the effective action for an $N=4$

\footnotetext{
*Present address: David Rittenhouse Laboratories, University of Pennsylvania, Philadelphia, Pennsylvania 19104. Email address: vijayb@pauli.harvard.edu

†Email address: egimon@ theory.caltech.edu

*Email address: minic@ physics.usc.edu

${ }^{\S}$ Email address: rahmfeld@theory.caltech.edu.

${ }^{1}$ See $[8,9]$ for reviews of conformal gravity.
}

super Yang-Mills (SYM) theory. This theory has a conformal anomaly [12] which reconstructs the action of $N=4$ conformal supergravity in four dimensions. By the AdS/CFT correspondence this must be related to a logarithmic divergent term of the spacetime action $[10,13]$. This implies that the asymptotically AdS solutions to $N=8$ gauged supergravity induce $N=4$ conformal gravity on the spacetime boundary. Turning this analysis around, the complete gravitational dynamics of the skin of the spacetime is reproduced holographically [14-16] by the conformal anomaly of the dual Yang-Mills theory, thus lending further support to the holographic renormalization group (RG) setup [17-22].

Conformal gravity remains one of the few classical theories of gravity that has not been integrated into the framework of string theory. The paper concludes with speculations about the role of conformal gravity, and discusses some applications of our results in the AdS/CFT and RandallSundrum contexts.

\section{ONE SUPERSYMMETRY FROM TWO}

The pure $N=2, d=5$ gauged supergravity [5] admits solutions that asymptotically have constant negative curvature. We seek the residual symmetries induced on the boundary of such spaces by the bulk theory. It will transpire that the boundary fields transform in multiplets of the $N=1, d=4$, conformal supergravity (listed in [8], Sec. 2.2). Maximally supersymmetric gauged supergravities in $d=3,6,7$ were related to conformal supergravities in $d=2,5,6$ in [23]. We will first present the fields and symmetries of the pure $N$ $=2$ gauged supergravity in five dimensions, and then argue that the boundary values have the correct multiplicities to form the gravity multiplet of 4D $N=1$ conformal supergravity. Finally, we show that the radial diffeomorphisms and supersymmetries of the bulk induce the symmetries of conformal supergravity on the four dimensional boundary. 


\section{A. $N=2$ gravity in five dimensions}

The gravity multiplet of the minimal gauged supergravity in five dimensions consists of the fünfbein $\hat{e} \hat{a}$, two gravitinos $\hat{\psi}_{\hat{\rho} i}$, and a gauge field $\hat{A}_{\hat{\mu}}$, where $i=1,2$. The gravitinos are related by the pseudo-symplectic Majorana condition. A $U(1)$ subgroup of the $S U(2)$ automorphism group of the $N=2$ algebra is gauged, and the field $\hat{A}_{\hat{\mu}}$ serves as the corresponding gauge field. The Lagrangian of the theory is then given (up to four-fermion terms) by [5]

$$
\begin{aligned}
& \hat{e}^{-1} \hat{\mathcal{L}}_{5}=-\frac{1}{2} \hat{R}-\frac{1}{2} \hat{\bar{\psi}}_{\hat{\mu}}^{i} \hat{\gamma}^{\hat{\mu}} \hat{\nu} \hat{\rho} \hat{\mathcal{D}}_{\hat{\rho}} \hat{\psi}_{\hat{\rho} i}-\frac{3 R^{2}}{32} \hat{F}_{\hat{\mu} \hat{\nu}} \hat{F}^{\hat{\mu} \hat{\nu}} \\
& -\frac{3 i}{4 R} \hat{\bar{\psi}}_{\hat{\mu}}^{i} \hat{\gamma}^{\hat{\mu} \hat{\nu}} \hat{\psi}_{\hat{\nu}}^{j} \delta_{i j}+\frac{6}{R^{2}} \\
& -\frac{3 i R}{32}\left(\hat{\bar{\psi}}_{\hat{\mu}}^{i} \hat{\gamma}^{\hat{\mu} \hat{\nu} \hat{\rho} \hat{\sigma}} \hat{\psi}_{\nu i} \hat{F}_{\hat{\rho} \hat{\sigma}}+2 \hat{\bar{\psi}} \hat{\mu} i \hat{\psi}_{i}^{\hat{\nu}} \hat{F}_{\hat{\mu} \hat{\nu}}\right) \\
& +\frac{\hat{e}^{-1}}{6 \sqrt{6}} c \hat{\epsilon} \hat{\mu} \hat{\nu} \hat{\rho} \hat{\sigma} \hat{\lambda} \hat{F} \hat{\mu} \hat{\nu} \hat{F} \hat{\rho} \hat{A^{A}} \hat{\lambda},
\end{aligned}
$$

where $c$ is a constant. Typically, $N=2$ theories that are obtained in 5D by compactification of $M$ theory on a CalabiYau threefold include additional hypermultiplets containing the moduli of the compact space, including the 5D dilaton. Likewise, the multiplets of the maximal $N=8$ gauged supergravity can be decomposed in terms of an $N=2$ gravity multiplet, along with some hypermultiplets and vector multiplets. All of these multiplets may be studied by methods similar to those used below to study the $N=2$ gravity multiplet.

The normalization is chosen so that the vacuum is $\operatorname{AdS}_{5}$ space with radius $R$ :

$$
d s^{2}=\frac{R^{2}}{r^{2}}\left(d x^{\mu} d x^{\nu} \eta_{\mu \nu}+d r^{2}\right)
$$

Here, $\mu=0,1,2,3$, and $r$ is the radial direction with the spacetime boundary at $r=0$. For reference, in the notation of [5] our conventions are $g=\frac{3}{4}, P_{0}=(4 / R) \sqrt{\frac{2}{3}}, \quad V_{1}=1, \quad h_{1}$ $=(R / 2) \sqrt{\frac{3}{2}}$ and $h^{1}=1 / h_{1}$. Carets denote 5D objects, so that $\hat{\mu}=0,1,2,3,4$, etc., and the signature is $(-++++)$. Also, the first part of the Latin alphabet is reserved for tangent space indices. The gauge covariant derivative is

$$
\left[\hat{\mathcal{D}}_{\hat{\mu}}(\hat{\omega}) \hat{\psi}_{\hat{\nu}}\right]^{i}=\hat{D}_{\hat{\mu}}(\hat{\omega}) \hat{\psi}_{\hat{\nu}}^{i}+\frac{4}{3} \hat{A}_{\hat{\mu}} \delta^{i j} \hat{\psi}_{\hat{\nu} j}
$$

in terms of $\hat{D}_{\hat{\mu}}(\hat{\omega})$, the standard covariant derivative:

$$
\hat{D}_{\hat{\mu}}(\hat{\omega}) \hat{\psi}_{\hat{\nu}}^{i}=\partial_{\hat{\mu}} \hat{\psi}_{\hat{\nu}}^{i}+\frac{1}{4} \hat{\omega} \hat{\omega}_{\hat{\mu}} \hat{b} \psi_{\hat{\nu}}^{i}
$$

Finally, $i, j$ indices are raised with the epsilon symbol: $\psi^{i}$ $=\epsilon^{i j} \psi_{j}, \epsilon^{12}=1$.
The local supersymmetry variations are

$$
\begin{aligned}
\hat{\delta} \hat{e}_{\hat{\mu}}^{\hat{a}}= & \frac{1}{2} \hat{\bar{\epsilon}}^{i} \hat{\gamma}^{\hat{a}} \hat{\psi}_{\mu i} \\
\hat{\delta} \hat{\psi}_{\hat{\mu}}^{i}= & \hat{\mathcal{D}}_{\hat{\mu}}(\hat{\tilde{\omega}}) \hat{\epsilon}_{i} \\
& +\frac{i R}{8}\left(\hat{\gamma}_{\hat{\mu}}^{\hat{\nu} \hat{\rho}}-4 \delta_{\hat{\mu}}^{\hat{\nu}} \hat{\gamma}^{\hat{\rho}}\right) \hat{\widetilde{F}}_{\nu \hat{\rho}} \hat{\epsilon}_{i} \\
& +\frac{i}{2 R} \hat{\gamma}_{\hat{\mu}} \delta^{i j} \hat{\epsilon}_{j} \\
\hat{\delta} \hat{A} \hat{\mu}= & \frac{i}{R} \hat{\bar{\psi}}_{\hat{\mu}}^{i} \epsilon_{i} .
\end{aligned}
$$

Here,

$$
\begin{aligned}
& \hat{\widetilde{\omega}}_{\hat{\mu} \hat{a} \hat{b}}=\hat{\omega}_{\hat{\mu} \hat{a} \hat{b}}-\frac{1}{4}\left(\hat{\bar{\psi}}_{\hat{b}}^{i} \hat{\gamma}_{\hat{\mu}} \hat{\psi}_{\hat{a} i}+2 \hat{\bar{\psi}}_{\hat{\mu}}^{i} \hat{\gamma}_{[\hat{b}} \hat{\psi}_{\hat{a}] i}\right) \\
& \hat{\tilde{F}}_{\hat{\mu} \hat{\nu}}=\hat{F}_{\hat{\mu} \hat{\nu}}+\frac{i \sqrt{6}}{4} \hat{\bar{\psi}}_{[\hat{\mu}}^{i} \hat{\psi}_{\hat{\nu}] i} .
\end{aligned}
$$

In order to study the boundary limit of these supersymmetry variations, we will also require the radial coordinate transformations, parametrized by $\xi^{r}$ :

$$
\begin{aligned}
& \hat{\delta}_{\hat{\xi}} \hat{e}_{\hat{\mu}}^{\hat{a}}=\hat{\xi}^{r} \partial_{r} \hat{e}_{\hat{\mu}}^{\hat{a}}+\partial_{\hat{\mu}} \hat{\xi}^{r} \hat{e}_{r}^{\hat{a}} \\
& \hat{\delta}_{\hat{\xi}} \hat{\psi}_{\hat{\mu}}^{i}=\hat{\xi}^{r} \partial_{r} \hat{\psi}_{\hat{\mu}}^{i}+\partial_{\hat{\mu}} \hat{\xi}^{r} \hat{\psi}_{r}^{i} \\
& \hat{\delta}_{\xi} \hat{A}_{\hat{\mu}}=\hat{\xi}^{r} \partial_{r} \hat{A}_{\hat{\mu}}+\partial_{\hat{\mu}} \hat{\xi}^{r} \hat{A}_{r} .
\end{aligned}
$$

In the boundary limit $(r \rightarrow 0)$, these equations will act as conformal transformations, which, together with the induced supersymmetries, will reproduce the symmetries of four dimensional conformal supergravity.

\section{B. Conformal supergravity multiplet}

To begin we must identify what we mean by the "boundary degrees of freedom" which enjoy the symmetries of 4D conformal gravity. The vacuum solution to the equations of motion of $5 \mathrm{D}$ gauged supergravity is $\mathrm{AdS}_{5}$ space, which is non-compact and only has a boundary in the conformal sense. In perturbation around this background, solutions to the equations of motion either vanish or diverge at infinity. (This is the familiar split into normalizable and nonnormalizable modes in the AdS/CFT correspondence [24].) Here we will argue that a similar split holds for the fully non-linear equations of motion. The boundary fields are identified as the finite residue that remains after removing the scaling divergence of non-normalizable bulk fields. Notably, the non-chiral fermion of the bulk theory loses half its components in the process and becomes chiral. In this way, the boundary values of bulk fields realize the gravity multiplet of $N=1, d=4$ conformal supergravity. 
To proceed, set $\hat{\psi}=0$ and $\hat{A}=0$. Then Fefferman and Graham [26] have shown that near the boundary (at $r=0$ ), a general solution to the equations of motion can be written as

$$
d s^{2}=\frac{R^{2}}{r^{2}}\left(d x^{\mu} d x^{\nu} g_{\mu \nu}+d r^{2}\right)
$$

where $g_{\mu \nu}=g_{\mu \nu}^{0}(x)+O\left(r^{2}\right)$. In the language of [24], the $O\left(1 / r^{2}\right)$ piece of the metric is the non-normalizable mode whose boundary value determines a conformal class of boundary metrics: the divergence at $r=0$ is removed by multiplying the metric by any function scaling as $r^{2}$ as $r \rightarrow 0$, giving $\Omega(x)^{2} g_{\mu \nu}^{0}(x)$ as the boundary value of the metric. Equivalently, different rates of approach to $r=0$ at different boundary positions $x$ yield a conformal factor $\Omega(x)^{2}$.

Hence, the radial diffeomorphisms (2.9) become conformal transformations of the boundary metric as $r \rightarrow 0$ provided

$$
\hat{\xi}^{r} \equiv r \lambda_{D} .
$$

Such diffeomorphisms "warp" surfaces homeomorphic to the AdS boundary in the radial direction, producing different conformal factors in the limiting procedure that yields the boundary metric [25]. The $1 / r^{2}$ radial dependence of the leading term in the bulk metric determines that the boundary metric $g_{\mu \nu}^{0}(x)$ has a conformal weight of 2. Similarly, we will see that the radial dependence of other bulk fields also determines their boundary conformal weight.

We choose $g_{\mu \nu}^{0}$ as the representative of the boundary conformal class, and partially fix the local symmetries. Following existing examples constructed by Nishimura and Tanii in $d=4,6,7[23]$, set

$$
\begin{aligned}
\hat{e}_{\mu}^{a}(r, x) & =\frac{R}{r} e_{\mu}^{a}(x)+O(1), \quad \hat{e}_{r}^{a}=e_{\mu}^{r}=0, \quad \hat{e}_{r}^{r}=\frac{R}{r}, \\
\hat{\psi}_{r}^{i} & =0, \\
\hat{A}_{r} & =0 .
\end{aligned}
$$

( $\underline{r}$ is the radial tangent space index.) We will examine the linearized bulk equations of motion for $\hat{A}_{\mu}$ and $\psi$ in the above background and gauge, and then argue that the nonlinear couplings between these fields and the metric do not modify the asymptotic scaling of solutions.

First examine the $r$ dependence of $\hat{A}_{\mu}$. Asymptotically, solutions to the equation of motion,

$$
\frac{1}{\sqrt{-\hat{g}}} \partial \hat{\mu}\left(\hat{g} \hat{\mu} \hat{\nu} \partial \hat{\nu} \hat{A}_{\rho}\right)=0,
$$

are found by choosing $\hat{A}_{\mu}$ to be independent of the radial direction,

$$
\hat{A}_{\mu}=A_{\mu},
$$

and solving the resulting four-dimensional equation of motion. The radial diffeomorphisms (2.11) with a dilatation parameter $\lambda_{D}$ as in Eq. (2.13) leave $A_{\mu}$ invariant. So we find that the gauge field has zero weight under the boundary conformal transformations induced by radial diffeomorphisms.

Next we turn to the gravitino. After using Eqs. (2.14) the four- and five-dimensional spin connections become

$$
\begin{aligned}
& \hat{\omega}^{a r}=-\frac{1}{r} e^{a}, \\
& \hat{\omega}^{a b}=\omega^{a b},
\end{aligned}
$$

and the covariant derivatives reduce to

$$
\begin{aligned}
& \hat{D}_{\mu}=D_{\mu}-\frac{1}{2 r} \gamma_{\mu} \gamma_{r} \\
& \hat{D}_{r}=\partial_{r} .
\end{aligned}
$$

To determine the decomposition of the gravitino we use the (linearized) gravitino equation of motion

$$
\hat{\gamma}^{\hat{\mu} \hat{\nu} \hat{\rho}} \hat{D}_{\hat{\nu}} \hat{\psi}_{\hat{\rho} i}-\frac{3 i}{2 R} \hat{\gamma}^{\hat{\mu} \hat{\nu}} \hat{\psi}_{\hat{\nu}}^{j} \delta_{i j}=0
$$

This equation reduces, after using Eqs. (2.19), to

$$
\gamma^{\mu \rho}\left(\delta_{i j} \partial_{r}-\delta_{i j} \frac{1}{r}-\frac{3 i}{2 r} \epsilon_{i j} \gamma_{5}\right) \hat{\psi}_{\rho j}+\gamma^{\mu \nu \rho} D_{\nu} \gamma_{5} \hat{\psi}_{\rho i}=0
$$

where $\gamma_{5} \equiv \gamma^{\underline{\underline{r}}}$ squares to 1 . Note that $\hat{\gamma}^{\mu}=\hat{e}_{a}^{\mu} \gamma^{a}=(r / R) \gamma^{\mu}$. These two equations can be diagonalized by introducing

$$
\hat{\Psi}_{\rho} \equiv \hat{\psi}_{\rho 1}+i \hat{\psi}_{\rho 2},
$$

which satisfies

$$
\gamma^{\mu \rho}\left(\partial_{r}-\frac{1}{r}-\frac{3}{2 r} \gamma_{5}\right) \hat{\Psi}_{\rho}+\gamma^{\mu \nu \rho} D_{\nu} \gamma_{5} \hat{\Psi}_{\rho}=0 .
$$

$\Psi$ is then decomposed into a chiral and an anti-chiral component with respect to $\gamma_{5}$ :

$$
\begin{aligned}
& \hat{\Psi}_{\rho}^{R} \equiv \frac{1}{2}\left(1-\gamma_{5}\right) \hat{\Psi}_{\rho} \\
& \hat{\Psi}_{\rho}^{L} \equiv \frac{1}{2}\left(1+\gamma_{5}\right) \hat{\Psi}_{\rho} .
\end{aligned}
$$

The dominating solution to Eq. (2.23) is then given by

$$
\hat{\Psi}_{\rho}^{R}=\left(\frac{2 R}{r}\right)^{1 / 2} \Psi_{\rho}^{R} .
$$

The radial dependence of $\hat{\Psi}_{\rho}^{R}$ combines with the radial diffeomorphisms (2.11) and (2.13) to give a conformal weight of $-1 / 2$ for the boundary value $\Psi_{\rho}^{R}$. 
It is important not to forget the constrained components of the gravitino $\hat{\Psi}_{\rho}^{L}$, as they enter the supersymmetry variations. Let

$$
\hat{\Psi}_{\rho}^{L}=(2 R r)^{1 / 2} \Phi_{\rho}^{L}
$$

then $\Phi_{\rho}^{L}$ satisfies the following equation:

$$
4 \gamma^{\mu \rho} \Phi_{\rho}^{L}=\gamma^{\mu \nu \rho}\left[D_{\nu} \Psi_{\rho}^{R}-D_{\rho} \Psi_{\nu}^{R}\right]
$$

This equation is solved by

$$
\Phi_{\rho}^{L}=\frac{1}{3} \gamma^{\nu}\left[D_{\nu} \Psi_{\rho}^{R}-D_{\rho} \Psi_{\nu}^{R}\right]+\frac{i}{12} \gamma_{5} \gamma^{\lambda} \epsilon_{\lambda \rho}^{\sigma \tau}\left[D_{\sigma} \Psi_{\tau}^{R}-D_{\tau} \Psi_{\sigma}^{R}\right]
$$

It only remains to argue that the asymptotic scalings and resulting four dimensional conformal weights are unchanged when the non-linear couplings between all the fields are accounted for. Also, one has to ensure that the Ansatz for the 5D metric in Eqs. (2.14) is consistent in the presence of non-trivial fields. After all, this was derived in [26] only for pure gravity with a cosmological constant.

First we turn to the vierbein. To see that the asymptotic behavior

$$
\hat{e}_{\mu}^{a}(x, r)=\frac{R}{r} e_{\mu}^{a}(x)+\text { subleading terms }
$$

is consistent even with non-trivial fields we study the scalings of terms in the bulk action. If the cosmological constant dominates in the boundary limit, the Fefferman-Graham analysis which yielded Eq. (2.30) will continue to hold. By definition, the cosmological constant is $r$ independent. Given the asymptotic form of the gravitino and gauge field that we have derived, it is easily verified that the kinetic, interaction and four-fermion terms of the $N=2$ gravity action [Eq. (2.1) and [5]] scale to zero at least as fast as $\sim r^{2}$ when $r \rightarrow 0$. Hence their contribution is subleading and the asymptotic scaling of the vielbein (or metric) survives the nonlinear interactions.

The same logic applies to the gravitino and the gauge field. With the scaling Ansätze we have made for all the fields, the leading behavior of the non-normalizable modes of the 5D gravitini is not altered when the full interaction terms are accounted for. Neither is the asymptotic behavior of the gauge field affected. Certainly, though, the non-linear terms in the equations of motion give rise to interactions between the various modes. This is precisely as expectedconformal supergravity is not a free theory.

We have shown how to extract the boundary values $\left(e_{\mu}^{a}, \Psi_{\mu}, A_{\mu}\right)$ of the $N=2$ gauged supergravity multiplet $\left(\hat{e}_{\hat{\mu}}^{\hat{a}}, \hat{\Psi}_{\hat{\mu}}^{i}, \hat{A} \hat{\mu}\right)$ and argued that these fields transform with specific weights under the induced conformal transformations of the spacetime boundary. In particular, although the bulk gravitino is non-chiral, its boundary value is chiral. In fact, $\left(e_{\mu}^{a}, \Psi_{\mu}, A_{\mu}\right)$ is precisely the gravity multiplet of $N$ $=1$ conformal supergravity. It remains to show that even the supersymmetries of this $N=1$ theory are induced on the spacetime boundary by the bulk transformations (2.5) $-(2.7)$.

\section{Conformal supergravity symmetries}

We have already shown that conformal transformations are induced on the spacetime boundary by bulk radial diffeomorphisms. To treat the induced supersymmetries (SUSY) we start with the fünfbein. Define $\hat{\epsilon}=\epsilon_{1}+i \epsilon_{2}$, and decompose the SUSY parameter $\epsilon$ according to its chirality under $\gamma_{5}$. Following the analysis of the 5D gravitino, the two components should have appropriate scaling factors, so that

$$
\hat{\boldsymbol{\epsilon}}=(2 R)^{1 / 2}\left(r^{-1 / 2} \epsilon^{R}, r^{1 / 2} \eta^{L}\right)
$$

with $\gamma_{5} \epsilon^{R}=-\epsilon^{R}, \quad \gamma_{5} \eta^{L}=\eta^{L}$. As the divergent piece, $\epsilon$ becomes the 4D supersymmetry parameter, whereas $\eta$ parametrizes special supersymmetries. To leading order in $r$, we derive, from Eq. (2.5),

$$
\begin{aligned}
\delta e_{\mu}^{a} & =\frac{R}{2 r} \hat{\bar{\epsilon}}^{i} \gamma^{a} \hat{\psi}_{\mu i} \\
& =\frac{R}{2 r} \hat{\bar{\epsilon}} \gamma^{a} \hat{\Psi}_{\mu} \\
& =\bar{\epsilon}^{R} \gamma^{a} \Psi_{\mu}^{R}+O(r) \\
& =-\frac{1}{2} \bar{\Psi}_{\mu} \gamma^{a} \epsilon+O(r),
\end{aligned}
$$

where we introduced Majorana spinors

$$
\chi=\left(\begin{array}{l}
\chi^{R} \\
\chi^{L}
\end{array}\right), \quad \text { with } \quad\left(\chi^{R}\right)^{*}=\epsilon \chi^{L},
$$

with $\epsilon=-i \sigma_{2}$. This is nothing but the standard variation of the vierbein in four dimensions. Note that $\eta$ decouples from the transformation of $e_{\mu}^{a}$.

Next, we turn to the gauge field. Using Eq. (2.7) gives

$$
\begin{aligned}
\delta A_{\mu} & =2 i\left(\bar{\Phi}_{\mu}^{L} \epsilon^{R}+\bar{\Psi}_{\mu}^{R} \eta^{L}\right) \\
& =-i\left(\bar{\Phi}_{\mu} \gamma_{5} \epsilon-\bar{\Psi}_{\mu} \gamma_{5} \eta\right),
\end{aligned}
$$

which agrees with the transformation law given in [8].

The analysis of the gravitino is more difficult since Eq. (2.6) is rather complicated. Fortunately, many terms drop out in the boundary limit. First of all, $\hat{\delta} \hat{\Psi} \sim r^{-1 / 2}$, so the term containing $F$ vanishes, because it scales as $r^{1 / 2}$. Also, the difference between $\hat{\omega}$ and $\hat{\omega}$ disappears, because the bilinears in the gravitino scale with a higher power of $r$. One is left with

$$
\hat{\delta} \hat{\psi}_{\hat{\mu}}^{i} \sim \hat{\mathcal{D}}_{\hat{\mu}}(\hat{\omega}) \hat{\epsilon}_{i}+\frac{i}{2 R} \hat{\gamma}_{\hat{\mu}} \delta^{i j} \hat{\epsilon}_{j}
$$

where 


$$
\left(\hat{\mathcal{D}}_{\hat{\mu}} \hat{\epsilon}\right)^{i}=\hat{D}_{\hat{\mu}} \hat{\epsilon}^{i}+\frac{3}{4} \hat{A}_{\hat{\mu}} \delta^{i j} \hat{\epsilon}_{j}
$$

With Eq. (2.22) this translates the chiral component of the gravitino to

$$
\delta \Psi^{R}=D_{\mu} \epsilon^{R}+\frac{3 i}{4} A_{\mu} \epsilon^{R}-\gamma_{\mu} \eta^{L}
$$

implying

$$
\delta \Psi^{L}=D_{\mu} \epsilon^{L}-\frac{3 i}{4} A_{\mu} \epsilon^{L}-\gamma_{\mu} \eta^{R}
$$

The last term in this expression has two origins. First, the relation between five- and four-dimensional covariant derivatives contains an extra term according to Eqs. (2.19). Second, it can be shown that this term gives the same contribution as the last term in Eq. (2.35).

We have shown in Eqs. (2.32), (2.37) and (2.34) that the 5D SUSY transformations reduce on the boundary to the residual transformations:

$$
\begin{gathered}
\delta e_{\mu}^{a}=-\frac{1}{2} \bar{\psi}_{\mu} \gamma^{a} \epsilon \\
\delta \Psi=\mathcal{D}_{\mu} \epsilon-\gamma_{\mu} \eta, \\
\delta A_{\mu}=i\left(\bar{\Psi}_{\mu} \gamma_{5} \eta-\bar{\Phi}_{\mu} \gamma_{5} \epsilon\right),
\end{gathered}
$$

where

$$
\mathcal{D}_{\mu} \epsilon=D_{\mu} \epsilon-\frac{3 i}{4} \gamma_{5} A_{\mu} \epsilon
$$

These are precisely the transformations of $d=4, N=1$ conformal supergravity. This agrees well with the results of [27] where it was found that the $\mathrm{AdS}_{5} \times \mathrm{S}^{5}$ superisometries reduce to superconformal transformations on the boundary of the AdS space.

\section{Summary}

We have shown that the gravity multiplet and symmetries of four-dimensional, $N=1$ conformal supergravity are induced on the boundary of solutions to pure $N=2$ gauged supergravity in five dimensions. Similarly, $N=2 k$ gauged supergravity in five dimensions can be related to 4-dimensional $N=k$ conformal supergravity. It is worth asking whether the induced action on the spacetime boundary respects the conformal gravity symmetries. This action is generally divergent and requires regulation. The regulator may be chosen to preserve Weyl invariance, yielding an induced conformal theory of gravity. We will argue that if Weyl invariance is explicitly broken by, say, putting in a radial cutoff as in the Randall-Sundrum scenario, the action of 4D Poincare gravity can be induced on the boundary.

\section{CONFORMAL YANG-MILLS AND CONFORMAL GRAVITY}

In order to study the action induced on the spacetime boundary, it is convenient to work within the AdS/CFT correspondence with relates $4 \mathrm{D}, N=4$ conformal gravity and $5 \mathrm{D}, N=8$ gauged supergravity [2]. The conventional Lagrangian for Yang-Mills theory on a curved manifold ( $L$ $=\sqrt{g} g^{m k} g^{n l} F_{m n} F_{k l}$ ) enjoys a generalization with local $\mathcal{N}$ $=4$ superconformal invariance (see the review [8] and the recent work of Liu and Tseytlin [9]). ${ }^{2}$ For a single $S U(N)$ vector multiplet $\left(A_{m}, \psi_{i}, X_{i j}\right),{ }^{3}$ the Lagrangian is

$$
\begin{aligned}
L_{\mathrm{SYM}}= & -\frac{1}{4}\left(e^{-\phi} F^{m n} F_{m n}+\mathcal{C} F^{m n} F_{m n}^{*}\right)-\frac{1}{2} \bar{\psi}^{i} \gamma^{m} D_{m} \psi_{i} \\
& -\frac{1}{4} X_{i j}\left(-D^{2}+\frac{1}{6} R\right) X^{i j} \\
& -X_{i j} F^{+m n} T_{m n}^{i j}+\mathcal{D}_{i j}^{k l}\left(X^{i j} X_{k l}-\frac{1}{6} \delta_{k}^{i} \delta_{l}^{i}|X|^{2}\right) \\
& +\cdots+\text { H.c. }
\end{aligned}
$$

The coupling constants $g_{m n}, \varphi=e^{-\phi}+i C$, etc., are in superconformal representations that fill out the field content of $\mathcal{N}=4$ conformal supergravity, but appear here as constant backgrounds rather than dynamical fields.

The effective action for the Euclidean field theory as a function of coupling constants is computed by integrating out the Yang-Mills fields, and gives a divergent part and a finite part:

$$
W\left(g_{m n}, \varphi, \ldots\right)=\int \mathcal{D} A \mathcal{D} \psi \mathcal{D} X e^{-S_{S Y M} \equiv W_{\mathrm{div}}+W_{\text {fin }}}
$$

The divergences that arise despite the conformal invariance of the theory are related to contact singularities in the definition of composite operators. Power law divergences can be cancelled by local counter-terms, but the effective action will contain a logarithmic divergence, $W_{\mathrm{div}}$, associated with the four dimensional conformal anomaly. ${ }^{4}$ Let us regulate this divergence by introducing a spatially uniform, covariant, Euclidean point-splitting cutoff: the end points of propagators in a Feynman diagram must be separated by a geodesic length exceeding some $\epsilon$. When $\epsilon$ is very small this can be written

$$
g_{m n}(\mathbf{x}) \Delta x^{m} \Delta x^{n} \geqslant \epsilon^{2} .
$$

\footnotetext{
${ }^{2}$ This section begins by summarizing standard results regarding conformal Yang-Mills theory coupled to conformal gravity, as transmitted to recent audiences by Liu and Tseytlin [9].

${ }^{3} i, j=1,2,3,4$ are indices of the $\mathrm{SU}(4) R$ symmetry.

${ }^{4}$ There are potential quartic and quadratic divergences which are proportional to an effective cosmological constant and the Ricci scalar of the manifold.
} 
$W_{\text {div }}$ diverges in the limit that $\epsilon$ vanishes. The difference in actions computed for $\nu$ vector multiplets, and with cutoffs $\epsilon$ and $\bar{\epsilon}$, is ${ }^{5}$

$$
\begin{aligned}
W_{d i v}= & \frac{\nu}{4(4 \pi)^{2}} \ln \left(\frac{\epsilon}{\bar{\epsilon}}\right) \int d^{4} x \sqrt{g} L_{\mathrm{CSG}} \\
L_{\mathrm{CSG}}= & C_{m n k l} C^{m n k l}-E+4\left[D^{2} \varphi^{*} D^{2} \varphi\right. \\
& \left.-2\left(R^{m n}-\frac{1}{3} g^{m n} R\right) D_{m} \varphi^{*} D_{n} \varphi\right]+\cdots
\end{aligned}
$$

Here $C$ is the Weyl tensor, $E$ is the Euler invariant, and $C^{2}$ $-E=2\left(R_{m n} R^{m n}-R^{2} / 3\right) . L_{\mathrm{CSG}}$ is precisely the Lagrangian for four dimensional $N=4$ conformal supergravity. [We will take $\nu=N^{2}-1 \approx N^{2}$ for an $S U(N)$ Yang-Mills theory at large $N$.] Integrating out the Yang-Mills fields has "induced" a Weyl invariant theory of gravity on the manifold.

Classically, the Lagrangian (3.2) is conformally invariant and is independent of the Weyl factor in the background metric-equivalently, the trace of the classical stress tensor vanishes. However, the logarithmic divergence in Eq. (3.6) results in an anomalous dependence on the Weyl factor of the metric and results in an anomalous trace in the stress tensor:

$$
T=\frac{\nu}{2(4 \pi)^{2}}\left(R_{m n} R^{m n}-\frac{1}{3} R^{2}+\cdots\right) .
$$

The ellipsis denotes terms that appear when the other couplings in Eq. (3.2) such as $\phi, C$, etc., are spatially varying. In fact, the right hand side of Eq. (3.7) is proportional to the conformal supergravity Lagrangian (3.6).

Accordingly, the finite part of the effective action $W$ will contain an anomalous piece that depends on the Weyl factor of the metric and whose variation produces the trace (3.7). Including the Weyl invariant piece $W_{\text {inv }}$ gives the finite part of the action:

$$
W_{\text {fin }}=W_{\text {anom }}+W_{\text {inv }} .
$$

The Weyl invariant piece will be a series in even powers of $\epsilon$ because the curvature invariants forming the metricdependent part of the action have dimension 2 :

$$
W_{\text {inv }}=W_{0}+\epsilon^{2} W_{2}+\epsilon^{4} W_{4}+\cdots .
$$

The higher order terms vanish as the cutoff is removed and are regularization scheme dependent.

The conformal anomaly on the other hand is essentially scheme independent, barring a term proportional to $\nabla^{2} R$ in Eq. (3.7) whose regularization-dependent coefficient we have set to zero. Having fixed this ambiguity, $W_{\text {anom }}$ has a

\footnotetext{
${ }^{5}$ See [9] and references therein.
}

diffeomorphism invariant, but non-local, expression whose Weyl variation produces the anomaly (3.7) (see $[12,29,25])$. However, splitting the metric into a Weyl factor and a reference background,

$$
g_{i j}=e^{2 \sigma_{g}^{-}}
$$

yields a local expression for the dependence of $W_{\text {anom }}$ on $\sigma$ $[12,9]:^{6}$

$$
\begin{aligned}
W_{\text {anom }}= & -\frac{\nu}{2(4 \pi)^{2}} \int d^{4} x \sqrt{\bar{g}}\left[\left(\bar{R}_{m n}^{2}-\frac{1}{3} \bar{R}^{2}+2 \bar{D}^{2}\right.\right. \\
& \left.\times \varphi^{*} \bar{D}^{2} \varphi+\cdots\right) \sigma+2 \bar{G}^{m n} \bar{D}_{m} \sigma \bar{D}_{n} \sigma+2 \bar{D}^{m} \sigma \\
& \left.\times \bar{D}_{m} \sigma \bar{D}^{2} \sigma+\left(\bar{D}^{m} \sigma \bar{D}_{m} \sigma\right)^{2}\right]
\end{aligned}
$$

Here $\bar{G}_{m n}$ is the Einstein tensor of $\bar{g}_{m n}$ and the terms linear in $\sigma$ in Eq. (3.11) are precisely the conformal supergravity action of the reference metric $\bar{g}_{i j}$. The trace of the stress tensor can be expressed in terms of $\sigma$ and $\bar{g}_{m n}$ as $T=$ $-\left.\left(e^{-4 \sigma} / \sqrt{\bar{g}}\right)(\delta W / \delta \sigma)\right|_{\sigma=0}$ which reproduces Eq. (3.7). In fact, the divergent part of the effective action and the quadratic part of anomalous piece can be conveniently combined into a single, closed-form, non-local action. ${ }^{7}$

The logarithmic divergence and the related finite anomalous term are exact at 1 loop for $\mathcal{N}=4$ Yang-Mills theory (see the references in [9]) and so we can reliably extrapolate the weak coupling results above to the large 't Hooft coupling, large $N$ Yang-Mills theory which should be related to a classical gauged supergravity. According to the AdS/CFT correspondence, $W$, the effective action of the Yang-Mills theory as a functional of sources is equal to the classical action of the bulk gauged supergravity as a functional of boundary data. So we have just shown that to leading (logarithmic) order 5D $N=8$ supergravity induces $N=4$ conformal supergravity on the spacetime boundary. However, as we have seen, the effective action also contains finite anomalous and Weyl invariant terms. According to AdS/CFT, these terms should together reconstruct the dynamics of five dimensional $N=8$ gauged supergravity.

Unfortunately, although parts of the finite, Weyl invariant part of the action $\left[W_{\text {fin }}\right.$ in Eq. (3.8) $]$ are fixed by the classic analyses of the conformal anomaly, $W_{\text {fin }}$ also contains terms that are not under control in the strong coupling limit. For example, the power series in $\epsilon$ in Eq. (3.9) could be greatly modified. So we will try to milk the anomaly for as much data as possible in reconstructing the bulk spacetime from field theory.

\footnotetext{
${ }^{6}$ Note that the standard Riegert action employed here is potentially problematic in dimensions $d>2$ [29].

${ }^{7}$ See $[12,29,9,25]$ and references therein.
} 


\section{A. Anomaly and the renormalization group}

The renormalization group studies the transformation of the effective action $W$ as a function of the cutoff $\epsilon$. The basic idea is that a change of the cutoff $\epsilon \rightarrow \epsilon^{\prime}$ is equivalent to a redefinition of the couplings $g \rightarrow g^{\prime}$ at fixed cutoff. The resulting effective variation of the couplings as a function of the cutoff is described by the RG equation.

For example, we will show that redefining the cutoff $\epsilon$ in Eq. (3.4) by a spatially varying factor $e^{\lambda(x)}$ is equivalent, to leading order in $\epsilon$, to re-scaling the metric by a Weyl factor:

$$
\begin{gathered}
g_{m n} \Delta x^{m} \Delta x^{n} \geqslant \epsilon^{2} e^{2 \lambda(x)} \Rightarrow \widetilde{g}_{m n} \Delta x^{m} \Delta x^{n} \geqslant \epsilon^{2} \\
\tilde{g}_{m n} \equiv e^{-2 \lambda(x)}\left[g_{m n}+\nabla_{m} V_{n}(\mathbf{x}, \boldsymbol{\epsilon})\right. \\
\left.+\nabla_{n} V_{m}(\mathbf{x}, \boldsymbol{\epsilon})\right] .
\end{gathered}
$$

Here we have permitted general $\epsilon$-dependent diffeomorphisms of the manifold generated by the vector field $V^{i}(\mathbf{x}, \epsilon)$ since these are symmetries of the theory.

When $\lambda$ is constant it is easy to show the equivalence in Eq. (3.12) for the leading terms in the effective actionnamely, the anomalous and logarithmic pieces. Re-scaling $\epsilon$ by $e^{\lambda}$ shifts the logarithmic divergent term (3.5) by $\lambda \nu / 4(4 \pi)^{2}$ times the conformal supergravity action. The same shift is produced in the $W_{\text {anom }}$ by re-scaling the metric by $e^{-2 \lambda}$. However, $W_{\mathrm{div}}$ is left invariant by a Weyl re-scaling of the metric as long as the other fields in the conformal gravity Lagrangian (3.6) are also re-scaled by their Weyl weights. For example, the scalar $\varphi$ has weight zero and therefore remains invariant. So when $\lambda$ is constant, a change in the cutoff can be traded, in the leading terms of the effective action, for a Weyl re-scaling of the metric and a corresponding re-scaling of all the couplings in Eq. (3.2) by their Weyl weights. However, the cutoff dependence of the Weyl invariant finite terms (3.9) implies that keeping the entire action invariant will require more than a Weyl transformation of the fields-in the perturbative limit, the couplings have to be corrected at each order in $\epsilon$ to keep the entire effective action invariant. These higher order corrections cannot be reliably extrapolated to the strong coupling limit, but non-renormalization of the conformal anomaly guarantees that as $\epsilon \rightarrow 0$, the equivalence (3.12) is valid.

It is a little harder to argue that this is still the case for a spatially varying cutoff, because the action (3.5) is explicitly computed for a constant cutoff. Instead, examine the origin of divergences and the anomaly in logarithmic singularities that occur when two points in a Feynman diagram approach each other closely. The cutoff in Eq. (3.12) restricts the proximity of such points by placing a lower bound on the size of vectors $\Delta X^{m}$ in the tangent space at $\mathbf{x}$. Re-scaling the cutoff increases the bound on $\Delta X^{m}$. Since the classical Lagrangian in Eq. (3.2) is both Weyl and diffeomorphism invariant, the Feynman diagrams are not changed by a combined re-scaling of the metric as in Eq. (3.13) and the other couplings by the appropriate Weyl weights. The only effect of this redefinition of couplings with a fixed small cutoff $\epsilon$ is to re-scale the bound on the size of vectors $\Delta X^{m}$ measuring separation between nearby points in the Feynman diagram integrations. In other words, at the level of the diagrammatic computation of the logarithmic divergent part of the effective action, a small spatially varying cutoff can be directly traded for a Weylrescaled metric as in Eq. (3.12). The anomalous terms in the action can be deduced from this following [12]. In this argument, it is essential that we understand the cutoff $\epsilon$ to be both small and slowly varying-Eq. (3.12) is a covariant cutoff only under these conditions.

We have just argued that that a small, slowly varying cutoff can be traded for a redefined metric in a way that leaves the sum of the logarithmic divergent and anomalous terms in the action invariant. The other couplings appearing in the effective action are also re-scaled according to their Weyl weights; e.g., the scalar $\varphi$ has weight 0 and remains invariant.

In fact, knowing the trace anomaly (3.7) of the theory fixes the anomalous part of the action and the logarithmic divergence up to Weyl invariant terms. First, it is possible to integrate the trace anomaly to find a diffeomorphism invariant action that varies to the anomaly $[12,29,25]$ (also see the references in [9]). This action is not unique - any local or non-local Weyl invariant may be added to it without changing the trace anomaly $[29,25]$ and other methods are required to determine these terms. Then, reversing the logic above we can infer the presence of a divergence logarithmic in the cutoff.

In essence, integrating out the Yang-Mills theory has "induced" a Weyl-invariant gravitational action on the manifold. According to the AdS/CFT correspondence, and in parallel with Sec. II, this action must also be induced by the bulk $N=8$ gauged supergravity. Below we will see how far we can go towards showing this directly from the bulk perspective.

\section{B. Gravity description}

Happily, the on shell massless fields of five dimensional $\mathcal{N}=8$ gauged supergravity have precisely the multiplicities of the couplings in Eq. (3.2), and transform in the same way under the asymptotic (super-conformal) symmetry group of the gravitational theory $[8,30]$. Following the AdS/CFT prescription we should compare the classical action for the 5-dimensional supergravity as a functional of boundary data to the Yang-Mills effective action $W$.

The cutoff length scale $\epsilon$ that appears in the field theory effective action is related to radial positions in the bulk space [15-19]. Indeed, when $\epsilon$ is small and slowly varying as a function of boundary positions $x$, it can be directly identified with a radial cutoff $[17,19,25]$. The field theory scheme dependence of choosing spatially varying cutoffs $\epsilon(x)$ is directly related to truncations of the bulk space by "wavy" surfaces parametrized as $r(x)=\epsilon(x)$ in the coordinates (2.12). All of these surfaces are related by five dimensional diffeomorphisms, and the metric induced on them is given precisely by Eq. (3.13) [25]. In other words, diffeomorphisms of the 5D spacetime are directly related to a choice of RG scheme for the dual field theory.

Henningson and Skenderis [10] showed that the gravitational terms in the 5D action contain quartic and quadratic 
divergences and a logarithmic divergence equal to the gravitational terms in Eq. (3.5). It was shown in $[10,11]$ that the power law divergences could be canceled by local boundary counterterms in the gravitational action. The leading piece of the bulk action as a functional of boundary data is then the logarithmically divergent term equal to the gravitational part of the four dimensional conformal anomaly. ${ }^{8}$ The results of [25] imply that this analysis continues to hold for a general foliation of the bulk spacetime by "wavy" cutoff surfaces. In other words, the leading gravitational terms in the five dimensional action exactly reproduce a conformally invariant action for boundary gravity as implied by the Yang-Mills conformal anomaly. In fact, these leading terms arise from the action accumulated by the divergent behavior of the metric near the boundary of the bulk space; finite energy excitations contribute subleading terms because, as implied by the results of [11], they contribute to finite parts of the action. So we learn that the complete gravitational dynamics of the skin of an asymptotically $\mathrm{AdS}_{5}$ space is contained the in the fourdimensional conformal anomaly.

The above discussion was carried out purely for the gravitational terms in the bulk and boundary actions. However, it is expected that inclusion of the scalars, fermions and gauge fields of 5D $N=8$ supergravity would induce the full $N=4$ conformal supergravity action on the boundary of the space. In the previous section we showed that $5 \mathrm{D}, N=2$ gauged supergravity induces the symmetries of $N=1$ superconformal gravity on the boundary. Here we expect (although it is technically much harder to show) that the symmetries of $N$ $=4$ conformal gravity are induced on the boundary. Given these symmetries, transformations of the gravitational terms $\left(C^{2}-E\right)$ are expected to give the remaining terms of the $N$ $=4$ conformal gravity Lagrangian.

\section{Summary}

We have used the AdS/CFT correspondence to argue that $N=4$ conformal gravity is induced on the 4-dimensional boundary of solutions to 5-dimensional $N=8$ gauged supergravity. Turning things around, we have also argued that the 4D conformal anomaly encapsulates the gravitational dynamics of the skin of asymptotically $\mathrm{AdS}_{5}$ spaces. Conformal gravities also exist in odd dimensions where there is no conformal anomaly. In these situations which arise, for example, in the $\mathrm{AdS}_{4} / \mathrm{CFT}_{3}$ correspondence, the bulk action does not have a logarithmic divergence and must induce a finite conformally invariant action on the boundary.

\section{DISCUSSION THE ROLE OF CONFORMAL GRAVITY}

To summarize, we have demonstrated, with an explicit mapping of symmetry actions, how $N=2$ gauged supergravity in the bulk of AdS space induces $N=1$ conformal supergravity on a boundary surface. The explicit breaking of Weyl symmetry involved in restricting to a cutoff surface will only add small perturbations to this scheme if we keep the surface

\footnotetext{
${ }^{8}$ In [28] this analysis was extended to dilatonic gravity.
}

near the boundary of AdS. With additional algebraic complexity a similar induction of $N=4$ boundary conformal supergravity should follow from bulk $N=8$ gauged supergravity. This is also well motivated from our discussion of the anomaly structure of $N=4$ SYM theory. Below we will argue that when we use boundaries far inside AdS, Weyl symmetry is strongly broken and the boundary theory is no longer conformal. In this situation, which is exploited in the Randall-Sundrum model, the induced gravity is only Poincaré invariant.

\section{A. Supersymmetric counterterms and holographic RG flows}

Our results regarding $N=2$ gauged supergravity have notable applications to the derivation of supersymmetric boundary counterterms for AdS gravity and holographic RG flows derived from the resulting regulated actions.

It has been shown that the power law divergences in the action and stress tensor of a space that is asymptotically locally AdS can be eliminated by the introduction of intrinsic boundary counterterms [11]. These methods avoid various ambiguities and technical difficulties associated with other methods in classical gravity for computing the action and conserved charges of a space. Using the induced $N=1$ SUSY boundary transformations that we have derived, one could compute the counterterms for the entire gravity supermultiplet by transforming the gravitational counterterms of [11].

Also, the AdS/CFT correspondence states that the classical action for the bulk space, regulated by these boundary counterterms and seen as a functional of boundary data, is equal to the effective action for an $N=1$ SYM theory that is conformal in the ultraviolet. This theory has a conformal anomaly which, in addition to the familiar Weyl tensor squared and Euler invariant contributions $\left(C^{2}-E\right)$, includes terms involving scalars and fermions. By the AdS/CFT correspondence, this must be equal to a logarithmic divergent term of the 5D spacetime action. Acting on $C^{2}-E$ with the explicit $N=1$ superconformal transformations that we have identified will yield the complete strong coupling supersymmetric conformal anomaly of the dual $N=1$ SYM theory.

In Sec. III A we discussed the matching between field theory cutoffs and radial positions in the bulk space. This is the basis of the holographic renormalization group [17-19]. By matching the bulk $N=2$ SUSY with the boundary $N$ $=1$ SUSY for the theory at each length scale of a given renormalization flow, our methods can also provide useful tools for the holographic analysis of Shifman-Vainshtein relations between supersymmetric beta functions.

\section{B. Induced Poincaré gravity: The $N=1$ supersymmetric Randall-Sundrum model}

Thus far we have discussed how Weyl-invariant gravity is induced on the boundary of spaces governed by gauged supergravity. Our considerations are also relevant to analyses of Randall-Sundrum models where the standard model is attached to a domain wall in five dimensional AdS space on which four dimensional Poincaré invariant gravity has been localized [4]. 
We showed in Sec. II that radial diffeomorphisms of five dimensional gauged supergravity induce Weyl transformations of the surfaces homeomorphic to the spacetime boundary. If Weyl symmetry is maintained as a residual symmetry on such surfaces, the induced gravitational action is conformally invariant (up to an anomaly). In other words, in the computation of the on-shell bulk supergravity action the counterterms [11], which make the bulk action finite, also precisely cancel induced boundary quantities that break the residual Weyl symmetry such as the Einstein-Hilbert and cosmological terms. Weyl invariance is nevertheless broken anomalously as in Sec. III by the radial cutoff dependence of a logarithmic divergence which cannot be canceled.

However, in situations like the Randall-Sundrum scenario [4], Weyl invariance is explicitly broken by the choice of a fixed radial position in AdS space where a brane is placed. In this case, there is no reason to pick a regulation scheme such as [11] which preserves the residual Weyl symmetry. Indeed, the Einstein-Hilbert counterterm in [11] can be ignored completely, allowing the bulk to induce Poincaré invariant gravity on fixed-radius surface. Then, the results of Sec. II can be used to study the $N=1$ supersymmetric structure induced by the bulk theory. However, the residual $N=1$ supersymmetry still forbids the appearance of a cosmological constant. This reasoning also applies to extended supersymmetry. An $N$ $=2 \mathrm{k}$ supersymmetric bulk supergravity extension of the Randall-Sundrum model will "induce" an $N=k$ Poincaré supergravity on the wall (see also [31]).

\section{Dynamical conformal gravity?}

In this paper we have discussed the appearance of conformal gravity at the boundary of spaces governed by gauged supergravity. The fields that appeared in this discussion were the boundary values of non-normalizable bulk modes that appear in asymptotically AdS spaces [24]. These modes cannot fluctuate unless the space is truncated at a finite radius, because their action is infinite if the bulk is non-compact. Therefore, the conformal gravity induced on the boundary of AdS space is not dynamical. Equivalently, as discussed in Sec. III, conformal gravity appears from the AdS/CFT perspective as the effective action of a field theory and is a functional of sources. Such effective actions do not describe dynamical theories-they are merely generating functions for correlators and should not be varied to compute equations of motion.

However, if the bulk space is cut off at a finite distance, the (formerly) non-normalizable modes that we have used to induce conformal gravity will have finite actions. Therefore they will be able to fluctuate. This suggests that they should become actual dynamical fields on the cutoff surface, and that dynamical conformal gravity is the effective theory observed by an experimentalist placed on a surface of fixed radius near the boundary of an asymptotically AdS space. ${ }^{9}$ A sigma model of closed strings in AdS space presumably includes world sheets with boundaries attached to the spacetime boundary. Such string configurations are the natural sigma model analogues of the non-normalizable modes. It would be natural to expect that they are responsible for inducing dynamical conformal gravity on surfaces near an AdS boundary.

The matter is subtler from the perspective of the dual CFT. In the large $N$ limit, we usually equate the SYM effective action with a path integral over bulk supergravity fields subject to boundary conditions on the AdS boundary. These boundary conditions are implemented by choosing a nonnormalizable mode background. Regulating the SYM theory is equivalent to cutting off the AdS space at some finite distance. At this cutoff surface, the values of supergravity fields are cannot be fully fixed and should be integrated over. Since boundary values of the supergravity modes correspond to sources in the SYM theory [24], it would seem that in the regulated SYM effective action we must integrate over both the fields and the sources, including the superconformal gravity multiplet. The resulting path integral is not a functional of the sources anymore, but rather a functional of the initial and final states. Hence, it would compute an $S$ matrix for the modified CFT. As the cutoff $\epsilon \rightarrow 0$ in this modified path integral, we require that the sources become frozen. This suggests an interesting perspective: the cutoff $\epsilon$ in some sense translates to an effective $\hbar$ for field theory sources within the AdS/CFT correspondence.

\section{ACKNOWLEDGMENTS}

We have enjoyed discussions with R. Corrado, B. de Wit, J. de Boer, P. Hořava, H. Ooguri, K. Pilch, L. Randall, S. Rey, K. Sfetsos, K. Stelle, E. Witten and especially A. Tseytlin. V.B. was supported by the Harvard Society of Fellows, the Milton Fund of Harvard University and by NSF grant NSF-PHY-9802709. E.G. and J.R. were supported by DOE grant DE-FG03-92ER40701 and the Caltech Discovery Fund. D.M. was supported by DOE grant DE-FG0384ER40168, and thanks the University of Illinois at Chicago for hospitality during the final part of this project.

\footnotetext{
${ }^{9}$ Dynamical gravity on the brane was also considered in [32].
}

[1] T. Kaluza, Sitzungsber. K. Preuss. Akad. Wiss., Phys. Math. K1. 1921, 996 (1921); O. Klein, Z. Phys. 37, 895 (1926).

[2] J. Maldacena, Adv. Theor. Math. Phys. 2, 231 (1998).

[3] S.S. Gubser, I.R. Klebanov, and A.M. Polyakov, Phys. Lett. B 428, 105 (1998); E. Witten, Adv. Theor. Math. Phys. 2, 253 (1998).

[4] L. Randall and R. Sundrum, Phys. Rev. Lett. 83, 3370 (1999);
83, 4690 (1999).

[5] M. Gunaydin, G. Sierra, and P.K. Townsend, Nucl. Phys. B253, 573 (1985); see also ibid. B242, 244 (1984).

[6] M. Pernici, K. Pilch, and P. van Nieuwenhuizen, Nucl. Phys. B259, 460 (1985); M. Gunaydin, L.J. Romans, and N.P. Warner, ibid. B272, 598 (1986).

[7] M.J. Duff and J.X. Lu, Phys. Lett. B 273, 409 (1991); G.T. 
Horowitz and A. Strominger, Nucl. Phys. B360, 197 (1991).

[8] E.S. Fradkin and A.A. Tseytlin, Phys. Rep. 119, 233 (1985).

[9] H. Liu and A.A. Tseytlin, Phys. Rev. D 59, 086002 (1999).

[10] M. Henningson and K. Skenderis, J. High Energy Phys. 07, 023 (1998).

[11] V. Balasubramanian and P. Kraus, Commun. Math. Phys. 208, 413 (1999).

[12] E.S. Fradkin and A.A. Tseytlin, Phys. Lett. 134B, 187 (1984); R.J. Riegert, ibid. 134B, 56 (1984).

[13] R. Emparan, C.V. Johnson, and R.C. Myers, Phys. Rev. D 60, 104001 (1999).

[14] G. 't Hooft, gr-qc/9310026; L. Susskind, J. Math. Phys. 36, 6377 (1995).

[15] L. Susskind and E. Witten, hep-th/9805114.

[16] A.W. Peet and J. Polchinski, Phys. Rev. D 59, 065011 (1999).

[17] V. Balasubramanian and P. Kraus, Phys. Rev. Lett. 83, 3605 (1999).

[18] E. Alvarez and C. Gomez, Nucl. Phys. B541, 441 (1999); E.T. Akhmedov, Phys. Lett. B 442, 152 (1998); C. Schmidhuber, Nucl. Phys. B580, 121 (2000).

[19] J. de Boer, E. Verlinde, and H. Verlinde, J. High Energy Phys. 08, 003 (2000); V. Balasubramanian, E. Gimon, and D. Minic, ibid. 05, 014 (2000).

[20] I.R. Klebanov and E. Witten, Nucl. Phys. B556, 89 (1999).

[21] D.Z. Freedman, S.S. Gubser, K. Pilch, and N.P. Warner, hep-th/9904017; J. High Energy Phys. 07, 038 (2000); L. Girardello, M. Petrini, M. Porrati, and A. Zaffaroni, ibid. 12, 022 (1998); Nucl. Phys. B569, 451 (2000).

[22] V. Sahakian, Phys. Rev. D 62, 126011 (2000).

[23] M. Nishimura and Y. Tanii, Mod. Phys. Lett. A 14, 2709 (1999); Int. J. Mod. Phys. A 14, 3731 (1999); M. Nishimura, hep-th/0004179; O. Coussaert, M. Henneaux, and P. van Driel, Class. Quantum Grav. 12, 2961 (1995); M. Banados, K. Bautier, O. Coussaert, M. Henneaux, and M. Ortiz, Phys. Rev. D 58, 085020 (1998); M. Henneaux, L. Maoz, and A. Schwimmer, Ann. Phys. (N.Y.) 282, 31 (2000).

[24] V. Balasubramanian, P. Kraus, and A. Lawrence, Phys. Rev. D 59, 046003 (1999); V. Balasubramanian, P. Kraus, A. Lawrence, and S.P. Trivedi, ibid. 59, 104021 (1999).

[25] C. Imbimbo, A. Schwimmer, S. Theisen, and S. Yankielowicz, Class. Quantum Grav. 17, 1129 (2000).

[26] C. Fefferman and C.R. Graham, Elie Cartan et les Mathematiques d'Aujourdhui, Asterisque, 1985, p. 95.

[27] P. Claus, J. Rahmfeld, H. Robins, J. Tannenhauser, and Y. Zunger, J. High Energy Phys. 07, 047 (2000); H. Ooguri, J. Rahmfeld, H. Robins, and J. Tannenhauser, ibid. 07, 045 (2000).

[28] S. Nojiri and S.D. Odintsov, Phys. Lett. B 444, 92 (1998).

[29] S. Deser, Phys. Lett. B 479, 315 (2000).

[30] For a review of the relevant group theory and references consult S. Ferrara and A. Zaffaroni, hep-th/9908163; D 'Hoker D. Freedman, and W. Skiba, Phys. Rev. D 59, 045008 (1999); M. Günaydin and D. Minic, Nucl. Phys. B523, 145 (1998); M. Günaydin, D. Minic, and M. Zagermann, ibid. B534, 96 (1998); B544, 737 (1999).

[31] J. Bagger, T. Moroi, and E. Poppitz, hep-th/0003282; E. Bergshoeff, R. Kallosh, and A. Van Proeyen, hep-th/0007044; T. Gherghetta and A. Pomarol, Nucl. Phys. B586, 141 (2000); N. Alonso-Alberca, P. Meessen, and T. Ortin, Phys. Lett. B 482, 400 (2000); A. Falkowski, Z. Lalak, and S. Pokorski, hep-th/0004093.

[32] M. Abou-Zeid, hep-th/0001127. 